\title{
On defect groups of the Mackey algebras for finite groups
}

\author{
Fumihito ODA
}

(Received June 2, 1996)

\begin{abstract}
In this paper, we introduce a new Mackey functor $\mathcal{T}$ and give a relation of ordinary defect group and defect group of the Mackey algebra of a finite group.
\end{abstract}

Key words: Mackey algebra, Mackey functor, group representation, block, defect group.

\section{Introduction}

The Mackey algebra $\mu_{R}(G)$ of a finite group $G$ over a commutative ring $R$ introduced by J. Thévenaz and P.J. Webb [TW] for studying the structure of Mackey functors. This is an algebra of finite rank over $R$ with the property that the category of Mackey functors of $G$ over $R$ is equivalent to the category of left $\mu_{R}(G)$-modules. So Thévenaz and Webb studied the blocks of Mackey functors in terms of the simple Mackey functors. In [TW] they determined the division of the simple Mackey functors into blocks of Mackey functors.

On the other hand, Yoshida introduced the span ring of the category of finite $G$-sets and gave the formula of the centrally primitive idempotents of the span ring [Yo]. It is interesting that the Mackey algebra $\mu_{R}(G)$ is isomorphic to the span ring of the category of finite $G$-sets. We can apply the formula of the span ring to the Mackey algebra $\mu_{R}(G)$. A centrally primitive idempotent of the span ring is indexed by the $p$-perfect subgroup $J$ and the $p$-block of $N_{G}(J) / J$. In particular, we consider that the $p$-blocks of the group algebra of $G$ is the corresponding centrally primitive idempotents of the span ring indexed by the trivial subgroup and $p$-blocks of $N_{G}(1) / 1=G$.

In this paper, we consider a defect group of the blocks of Mackey functors of $G$ like as the ordinary block theory. The word "blocks of Mackey functors" means two-sided direct summands of $\mu_{R}(G)$ or the corresponding centrally primitive idempotents of $\mu_{R}(G)$. We introduce a Mackey functor $\mathcal{T}$ for the sake of the definition of a defect groups of blocks of Mackey functors. The inductions of $\mathcal{T}$ are generalization of the trace maps of the group

1991 Mathematics Subject Classification : Primary 20C20; Secondary 19A22, 20J05, $16 \mathrm{G} 20$. 
algebra as a $G$-algebra. We can give the definition a defect group of $\mu_{R}(G)$ using a Mackey functor $\mathcal{T}$ and study the relation of the group algebra.

The author wish to thank A. Watanabe for helpful suggestions concerning a Mackey functor $\mathcal{T}$ and $\mathrm{T}$. Yoshida for kind advice concerning the formula of the centrally primitive idempotents of the span ring and the theory of Mackey functors.

\section{Mackey functor $\mathcal{T}$}

In this section, we introduce a new Mackey functor $\mathcal{T}$ for a finite group $G$. Let $R$ be a commutative ring and $\mu_{R}(G)$ a Mackey algebra over $R$. Let $\alpha$ be an $R$-algebra homomorphism

$$
\alpha: \mu_{R}(H) \longrightarrow \mu_{R}(G)
$$

which is terms of symbols are $\alpha\left(I_{K}^{J}\right)=I_{K}^{J}, \alpha\left(R_{K}^{J}\right)=R_{K}^{J}$ and $\alpha\left(c_{h}^{K}\right)=c_{h}^{K}$ for all subgroups $K \leq J \leq H \leq G$, and $h \in H$. In general, $\alpha$ is not injective.

For a subgroup $H$ of $G$, we put

$$
\mu_{R}(G)_{H}=\left\{x \in \mu_{R}(G) \mid \alpha(\theta) x=x \alpha(\theta) \text { for all } \theta \in \mu_{R}(H)\right\} .
$$

Moreover, we put

$$
\mathcal{T}(H):=\mu_{R}(G)^{H}:=1_{H} \mu_{R}(G)_{H}
$$

where

$$
1_{H}=\sum_{L \leq H} I_{L}^{L} \in \mu_{R}(H)
$$

In particular, $\mathcal{T}(G)$ is the center of $\mu_{R}(G)$.

For all subgroups $K \leq H \leq G$ and $g \in G$ we define the $R$-homomorphisms $\mathcal{I}_{K}^{H}, \mathcal{R}_{K}^{H}, \mathcal{C}_{g}^{H}$ as follows:

$$
\begin{aligned}
& \mathcal{I}_{K}^{H}: \mathcal{T}(K) \rightarrow \mathcal{T}(H): \theta \mapsto \sum_{L \leq H} \sum_{h \in[L \backslash H / K]} I_{L \cap^{h} K}^{L} c_{h}^{L^{h} \cap K} \theta c_{h^{-1}}^{L \cap^{h} K} R_{L \cap^{h} K}^{L}, \\
& \mathcal{R}_{K}^{H}: \mathcal{T}(H) \rightarrow \mathcal{T}(K): \theta \mapsto 1_{K} \theta, \\
& \mathcal{C}_{g}^{H}: \mathcal{T}(H) \rightarrow \mathcal{T}\left({ }^{g} H\right): \theta \mapsto \sum_{L \leq{ }^{g} H} c_{g}^{L} \theta c_{g^{g}}{ }^{-1} .
\end{aligned}
$$

Proposition 1 Let $\mathcal{T}$ be as above notation with morphisms $\mathcal{I}, \mathcal{R}, \mathcal{C}$. Then $\mathcal{T}$ is the multiplicative Mackey functor (Green functor) for $G$. 
Proof. We only check the Mackey decomposition formula. For an element $\theta$ of $\mu_{R}(G)^{K}$, we have

$$
\begin{aligned}
\mathcal{R}_{J}^{H} \mathcal{I}_{K}^{H}(\theta) & =\sum_{E \leq J} I_{E}^{E} \sum_{L \leq H} \sum_{g \in[L \backslash H / K]} I_{L \cap{ }^{g} K}^{L} c_{g}^{L^{g} \cap K} \theta c_{g^{-1}}^{L \cap^{g} K} R_{L \cap g}^{L} K \\
& =\sum_{E \leq J} \sum_{g \in[E \backslash H / K]} I_{E \cap{ }^{g} K}^{E} c_{g}^{E^{g} \cap K} \theta c_{g^{-1}}^{E \cap^{g} K} R_{E \cap g}^{E} .
\end{aligned}
$$

On the other hand, for an element $\theta$ of $\mu_{R}(G)^{K}$

$$
\begin{aligned}
& \sum_{x \in[J \backslash H / K]} \mathcal{I}_{J \cap^{x} K}^{J} \mathcal{C}_{x}^{J^{x} \cap K} \mathcal{R}_{J^{x} \cap K}^{K}(\theta) \\
= & \sum_{x \in[J \backslash H / K]} \mathcal{I}_{J \cap^{x} K}^{J}\left(\sum_{E \leq J^{x} \cap K} c_{x}^{E} \theta c_{x^{-1}}^{x}\right) \\
= & \sum_{x \in[J \backslash H / K]} \sum_{L \leq J} \sum_{g \in\left[L \backslash J / J \cap^{x} K\right]} I_{L \cap^{g}\left(J \cap^{x} K\right)}^{L} c_{g} c_{x} \theta c_{x^{-1}} c_{g^{-1}} R_{L \cap^{g}\left(J \cap^{x} K\right)}^{L} I_{L}^{L} I_{L \cap^{g x} K} c_{g x} \theta c_{(g x)^{-1}} R_{L \cap^{g x} K}^{L} . \\
= & \sum_{L \leq J} \sum_{g x \in[L \backslash H / K]}
\end{aligned}
$$

In the next result we will see the fact that $\mathcal{T}$ is the generalization of fixed point functor of a group algebra $R G$. Let $H \leq G$, the $F P_{R G}(H)=R G^{H}$ is a fixed point set of $H$ in $R G$, i.e.,

$$
R G^{H}=\left\{x \in R G \mid h x h^{-1}=x, h \in H\right\} .
$$

Restriction, induction, conjugation are

$$
\begin{aligned}
& \operatorname{res}_{K}^{H}: R G^{H} \hookrightarrow R G^{K}: \text { embedding, } \\
& \operatorname{ind}_{K}^{H}: R G^{K} \rightarrow R G^{H}: x \mapsto \sum_{h \in[H / K]} h x h^{-1}, \\
& \operatorname{con}_{g}^{H}: R G^{H} \rightarrow R G^{g} H: x \mapsto g x g^{-1}
\end{aligned}
$$

where $K \leq H, g \in G$. Then we denote by $F P_{R G}$ the fixed point functor of $R G$. We remark that for a subgroup $H$ of $G$, there is a surjective homomorphism

$$
\pi_{H}: \mu_{R}(G)^{H} \rightarrow R G^{H}: \theta \mapsto I_{1}^{1} \theta R_{1}^{1} .
$$

Remark. Let $\mathcal{T}$ be as above notation. For subgroups $K \leq H$ and $g \in G$, 
we have

$$
\operatorname{ind}_{K}^{H} \pi_{K}=\pi_{H} \mathcal{I}_{K}^{H}
$$

\section{Defect group}

In general, a Mackey functor $M$ is projective relative to $\mathcal{X}$ if and only if the sum of inductions

$$
\theta_{\mathcal{X}}(G):=\sum_{H \in \mathcal{X}} t_{H}^{G}: \bigoplus_{H \in \mathcal{X}} M(H) \longrightarrow M(G)
$$

is surjective and split. However, Dress assert that if $M$ is a Green functor, then we need only to see that $\theta_{\mathcal{X}}$ is surjective [Dr] [Th] (2.4).

If $M$ is a Mackey functor for $G$ then there exists a unique minimal subconjugacy closed set $\mathcal{X}$ of $G$ such that $M$ is projective relative to $\mathcal{X}$. Dress called it the defect base of $M[\mathrm{Gr}],[\mathrm{Dr}]$, [Th].

Let $A$ be a multiplicative Mackey functor such that each algebra $A(H)$ is associative and has identity element $1_{H}$. Then the defect base of $A$ is the union of the defect base of Mackey functor $e_{i} A e_{i}(1 \leq i \leq n)$ for $G$ where

$$
e_{i} A e_{i}(H):=R_{H}^{G}\left(e_{i}\right) A(H) R_{H}^{G}\left(e_{i}\right) \quad\left(H \leq G, 1_{G}=e_{1}+\cdots+e_{n}\right),
$$

$e_{i}$ 's are mutually orthogonal idempotents. If the $e_{i}$ 's are centrally primitive idempotents then the defect base of $e_{i} A e_{i}$ is $\left\{Q_{i}\right\}$ (up to $G$-conjugacy), we say that $Q_{i}$ is the defect group of $e_{i} A e_{i}$.

From the formula for the centrally primitive idempotent of the Mackey algebra $\mu_{R}(G)$ (the span $\operatorname{ring} R S p\left(\mathcal{S}_{f}^{G}\right)$ [Yo] Lemma 3.4) we obtain the defect base of $\mathcal{T}$ and the defect group of $\mathcal{T}_{S, B}:=E_{S, B} \mathcal{T} E_{S, B}$. We call it the defect group of block idempotent $E_{S, B}$ of the Mackey algebra $\mu_{R}(G)$.

Theorem 2 Let $B$ be a p-block of $R G$ and $D$ the defect group of $B$ and let $P$ be the defect group of $E_{1, B}\left(\right.$ or $\left.\mathcal{T}_{1, B}\right)$. Then

$$
D \leq_{G} P \text {. }
$$

Proof. Let $e_{B}$ be the block idempotent of $B$. By the formula for the centrally primitive idempotent of the Mackey algebra [Yo] we can define the homomorphism $\pi_{G}$ (resp. $\pi_{P}$ ) of $\mathcal{T}_{1, B}(G)\left(\operatorname{resp} . \mathcal{T}_{1, B}(P)\right)$ to $F P_{e_{B} R G}(G)$ (resp. $\left.F P_{e_{B} R G}(P)\right)$. By Remark we can consider the next commutative 
square

$$
\begin{array}{ccc}
F P_{e_{B} R G(P)} & \stackrel{\operatorname{ind}_{P}^{G}}{\longrightarrow} & F P_{e_{B} R G(G)} \\
\uparrow \pi_{P} & & \uparrow \pi_{G} \\
\mathcal{T}_{1, B}(P) & \stackrel{\mathcal{I}_{P}^{G}}{\longrightarrow} & \mathcal{T}_{1, B}(G)
\end{array}
$$

where $\pi_{G}$ (resp. $\left.\pi_{P}\right)$ is restriction of $\mu_{R}(G)^{G}\left(\operatorname{resp} . \mu_{R}(G)^{P}\right)$ to $\mathcal{T}_{1, B}(G)$ (resp. $\left.\mathcal{T}_{1, B}(P)\right)$. By assumption, $\mathcal{I}_{P}^{G}$ is surjective and $\pi_{G}$ so is, ind ${ }_{P}^{G}$ is surjective. Hence, $F P_{e_{B} R G}$ is projective relative to $P$ from Remark and Dress's result [Dr], [Th] (2.4). Thus $D$ is contained in $P$ (up to conjugacy) by the minimality of the defect group of $B$.

\section{References}

[Dr] Dress A., Contributions to the theory of induced representations In: Algebraic Ktheory II. Proc. Batelle Institute Conference 1972 (Ed. H. Bass) Lecture Notes in Mathematics 342, 183-240, Springer-Verlag 1973.

[Gr] Green J.A., Axiomatic representation theory for finite groups. J. Pure Appl. Algebra 1, (1971), 41-77.

[Th] Thévenaz J., A visit to the kingdom of Mackey functors. Bayreuther Math. Schriften 33 (1990), 215-241.

[TW] Thévenaz J. and Webb, The structure of Mackey functors. Trans. A.M.S. 347 (1995), 1865-1961.

[Yo] Yoshida T., Idempotents and transfer theorems of Burnside rings, character rings and span rings. Algebraic and Topological Theories (to the memory of T. Miyata) (1985), 589-615.

Department of Mathematics

Hokkaido University

Sapporo 060, Japan 